\title{
Nuts and bolts of supersymmetry
}

\author{
Nitin Upadhyaya $\odot{ }^{1,2,3}$ Bryan G. Chen, ${ }^{4}$ and Vincenzo Vitelli ${ }^{1,2,5, *}$ \\ ${ }^{1}$ James Franck Institute, University of Chicago, Chicago, Illinois 60637, USA \\ ${ }^{2}$ Department of Physics, University of Chicago, Chicago, Illinois 60637, USA \\ ${ }^{3}$ Center for Mathematical Modeling, Flame University, Pune, Maharashtra 412115, India \\ ${ }^{4}$ Lorentz Institute for Theoretical Physics, Leiden University, Leiden 2333 CA, Netherlands \\ ${ }^{5}$ Kadanoff Center for Theoretical Physics, University of Chicago, Chicago, Illinois 60637, USA
}

(Received 29 June 2020; accepted 18 September 2020; published 19 October 2020)

\begin{abstract}
A topological mechanism is a zero-elastic-energy deformation of a mechanical structure that is robust against smooth changes in system parameters. Here, we map the nonlinear elasticity of a paradigmatic class of topological mechanisms onto a supersymmetric field theory introduced by Witten and Olive. Heuristically, this approach entails taking the square root of a nonlinear Hamiltonian. It generalizes the standard procedure of obtaining two copies of the Dirac equation by taking the square root of the linear Klein-Gordon equation. Our real-space formalism goes beyond topological band theory by incorporating nonlinearities and spatial inhomogeneities, such as domain walls (i.e., kinks), where topological states are typically localized. We interpret the two components of the real fermionic field as site and bond displacements, respectively. The constraint of zero elastic energy insures that kinks in the mechanical system saturate the Bogomolny-Prasad-Sommerfield bound, while forbidding antikinks. This mechanism can be viewed as a manifestation of the underlying supersymmetry being half broken.
\end{abstract}

DOI: 10.1103/PhysRevResearch.2.043098

\section{INTRODUCTION}

Mechanisms are deformations of a mechanical structure which cost zero elastic energy [1-5]. As an example, consider the folding motion of networks of bars or plates constrained by pivots or hinges around which two adjacent components can freely rotate. When actuated by intrinsic noise or motors and other external fields, such mechanisms could mimic selfpropelled motion [6] and become the building blocks of robots and smart metamaterials $[7,8]$ and display nonreciprocal elasticity [9]. Thus, the hard problem of predicting the effect of constraints on an interacting many-body system is as deeply rooted in mechanical design and robotic control theory as it is in modern theoretical physics [10,11].

Here, we study a special class of mechanisms called topological mechanisms which arise through an intriguing correspondence between the bulk and the boundary (or defects) of periodic mechanical structures on the verge of stability [12]. Such mechanisms are robust to smooth changes in material parameters so long as the global connectivity of the structure is preserved [12-19]. Inspired by the study of electronic topological materials [20-23], topological mechanical states are now being engineered that not only display many of

\footnotetext{
*vitelli@uchicago.edu

Published by the American Physical Society under the terms of the Creative Commons Attribution 4.0 International license. Further distribution of this work must maintain attribution to the author(s) and the published article's title, journal citation, and DOI.
}

the features originally thought to be exclusively in the domain of quantum condensed matter, but also provide novel ideas and phenomena, often using easy to assemble components such as Lego blocks [24-45].

Unlike their electronic counterparts, topological mechanisms are not adequately addressed by the theory of linear vibrations for the following reasons. First, mechanisms often involve large deformations of the mechanical structure and hence nonlinearities become paramount. While Maxwell's constraint counting theory applied to the linear vibrational spectrum can predict the presence of mechanisms, it does not describe their properties [46]. Second, topological mechanisms can exist in structures that are not periodic [39]. For example, domain walls or kinks that cost zero stretching energy can propagate through a one-dimensional topological [16] chain even in the presence of strong disorder [17], provided that no bonds are broken or states of self-stress created.

In this article, we propose a supersymmetric (SUSY) extension of nonlinear continuum mechanics that allows one to a priori keep track of internal degrees of freedom and deformations that inevitably accompany the dynamics of extended classical excitations, such as kinks and solitons. We illustrate this idea using the quasi-one-dimensional topological mechanism as a paradigmatic example of a classical system whose kink solution saturates the Bogomolny-Prasad-Sommerfield (BPS) bound [47-49]. Our approach consists of mapping the one-component boson (described by a nonlinear KleinGordon theory) to a two-component Majorana field (Dirac equation with real solution) via Dirac's square root procedure. We identify the square root of the Hamiltonian with one of the conserved charges in the Witten-Olive supersymmetric 
(SUSY) field theory, a proposed spacetime symmetry which relates bosons and fermions [50-55], and in the process, obtain another conserved charge, which we associate with a partner Hamiltonian. In the BPS saturated case, only one of these charges is zero, and therefore the supersymmetry is half broken. Further, we show that the two components of the Majorana field physically correspond to the kink-induced displacement and stress fields, respectively. Upon linearization, the half-broken supersymmetry results in an asymmetry between site displacements and bond extensions.

\section{LINEAR TOPOLOGICAL MECHANICS}

The first step toward studying mechanisms in an arbitrary mechanical structure is to identify (within linear theory) the zero-energy eigenvalues (modes) of the Fourier-transformed rigidity matrix (or equivalently dynamical matrix; see Appendix B), which within linear order relates bond stretching to site displacements [56]. Physically, a zero mode causes no stretching of the elastic bonds even when some of the sites are displaced. Conversely, a state of self-stress is an assignment of bond tensions that does not result in site displacements. The generalized Maxwell-Calladine relation [57] stipulates that for $N$ sites in $d$ dimensions, the number of zero modes $n_{\mathrm{m}}$ minus states of self-stress $n_{\mathrm{ss}}$ equals the number of degrees of freedom $N_{\mathrm{df}}=d N$ minus constraints $N_{\mathrm{c}}$,

$$
v \equiv n_{\mathrm{m}}-n_{\mathrm{ss}}=N_{\mathrm{df}}-N_{\mathrm{c}} .
$$

A structure is rigid, floppy, or isostatic, depending on whether $v<0, v>0$, or $v=0$, respectively [1,58,59]. Once the connectivity is fixed [which fixes the right-hand side of Eq. (1)], the index $v$ can be viewed as a topological charge [60], invariant under smooth deformations of the local bond length. Kane and Lubensky laid the foundations for the use of topological band theory to establish the topological nature and location of zero modes associated with so called Maxwell lattices [12]. While the study of mechanical topological modes began with phonons and, hence, linear vibrations in mind, zero modes could be either infinitesimal or finite motions of the structure and only a nonlinear analysis can reveal their full nature $[16,61]$.

\section{NONLINEAR MECHANICS OF TOPOLOGICAL CHAINS}

Before discussing the supersymmetric field theory, we briefly review the relation between zero-energy modes and nonlinear mechanisms for a quasi-one-dimensional mechanism; see Fig. 1 [12,16]. This realization consists of a periodically repeating, dimerized unit of green rigid rotors (alternately pointing up and down), each of length $r$ separated by a distance $a$ and constrained to rotate about fixed white bolts. The local orientation of each rotor with respect to the vertical is denoted by $\theta(x)$ and their horizontal projection by $u(x)=r \sin \theta(x)$. The rigid rotors are coupled to their nearest neighbors through orange bars that can be viewed as Hookean springs with elastic constant $k \rightarrow \infty$. There is exactly one fewer constraint than degrees of freedom in the chain; thus by Eq. (1) there is exactly one zero-energy mode [62]. If we denote by $\bar{\theta}$ the angle that the rotors make with the vertical

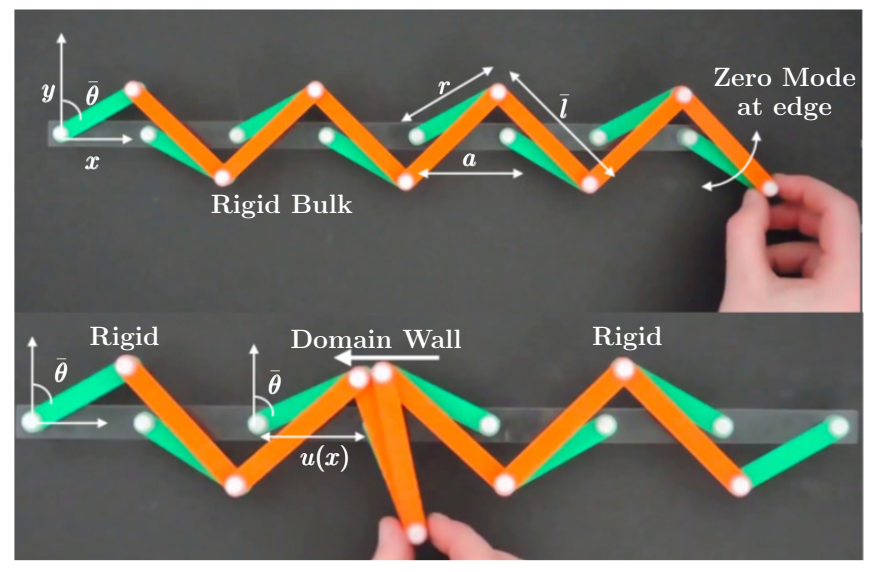

FIG. 1. A mechanism inspired by the organic molecule polyacetylene is constructed from rigid (green) rotors coupled by (orange) bars (see movies in Supplemental Material [71]). Once actuated (by hand here), the zero-energy mode travels down the chain (indicated by arrows). A domain wall separates the left and right leaning green bars. Here, $r$ is the length of the rotor, $a$ is the lattice spacing, $\theta(x)$ is the angle that the rotor at position $x$ makes with respect to the vertical, and $u(x)=r \sin \theta(x)$ is the projection of the rotor length along the $x$ axis.

in the initial uniform configuration (assumed positive in the clockwise sense), then the zero-energy mode will be localized to the left edge if $\bar{\theta}<0$ or right edge if $\bar{\theta}>0$.

In order to derive the continuum theory, we express the length $l$ of the rigid bar that connects two adjacent rotors in terms of $r, a$ and their respective angular displacements and solve for the rigid-bar constraint, i.e., $l=\bar{l}$, where $\bar{l}$ is the equilibrium length of the orange bars in the uniform state where $\theta(x)=\bar{\theta}, \pi-\bar{\theta}$; see Fig. 1 and Appendix B. In the limit that $2 r \sin \bar{\theta} \ll a$ and $a \ll 1$, we find the following nonlinear differential equation for $u(x)=r \sin \theta(x)$ [16]:

$$
\frac{a^{2}}{2} \frac{d u}{d x}=u^{2}-\bar{u}^{2},
$$

where $\bar{u}=r \sin \bar{\theta}$. The solution of this nonlinear differential equation (up to a constant) is a kink (domain wall)

$$
u_{s}=-\bar{u} \tanh \left(\frac{x-x_{0}}{\frac{a^{2}}{2 \bar{u}}}\right),
$$

which interpolates between the two topologically distinct uniform states of the chain $u(x \rightarrow \infty)=-\bar{u}$ and $u(x \rightarrow-\infty)=$ $\bar{u}$. Moreover, the kink can translate along the chain by a sequential activation of the joints; see Fig. 1. The dynamics is described by relaxing the rigid-bar constraint and introducing a finite spring constant $k$ (for orange bars) to obtain the nonlinear Hamiltonian [16]

$$
\mathcal{H}=\frac{H}{\rho}=\frac{1}{2} \int d x\left[\pi^{2}+c^{2}\left(\frac{\partial u}{\partial x}+\sqrt{V(u)}\right)^{2}\right],
$$

where we have rescaled the original Hamiltonian by the mass density $\rho=\frac{M}{a}$ ( $M$ being the mass of the rotors) and defined the conjugate momentum field $\pi(x, t)=\frac{\partial u}{\partial t}$, linear sound 


$$
\text { speed } c=\frac{a^{2}}{l} \sqrt{\frac{k}{M}} \text {, and }
$$

$$
V(u)=\frac{4}{a^{4}}\left(u^{2}-\bar{u}^{2}\right)^{2} .
$$

Note that the Hamiltonian in Eq. (4) is the sum of two perfect squares. Consequently, the static kink configuration in Eq. (3) that solves the first-order constraint equation (2) can be simply obtained by setting to zero the term within round brackets in Eq. (4).

The approach we adopt was first proposed by Bogomolny $[47,48]$. This method that we briefly review is used to directly obtain first-order equations that yield static kink (and antikink) configurations of the field (without finding the equations of motion). It is instructive to compare Eq. (4) to a Hamiltonian of the form

$$
\mathcal{H}=\frac{1}{2} \int d x\left[\pi^{2}+\left(\frac{\partial u}{\partial x}\right)^{2}+V(u)\right] .
$$

If a double-well potential of the form in Eq. (5) is chosen, Eq. (6) describes an Ising model. Upon completing the square one obtains

$$
\begin{aligned}
\mathcal{H}= & \frac{1}{2} \int d x\left[\pi^{2}+\left(\frac{\partial u}{\partial x} \mp \sqrt{V(u)}\right)^{2}\right] \\
& \pm \int d u \sqrt{V(u)} .
\end{aligned}
$$

When $c=1$, Eq. (7) reduces to Eq. (4) aside from the last term in Eq. (7) which, as we shall see, is a boundary term. If $u(x \rightarrow \infty)= \pm \bar{u}$, then energy is minimized if

$$
\pi=0, \quad \frac{\partial u}{\partial x} \mp \sqrt{V(u)}=0 .
$$

The minimum energy $E$ of the corresponding field configuration is then given by

$$
E= \pm \int d u \sqrt{V(u)}
$$

where the plus and minus signs correspond to static kink and antikink solutions, respectively. It is convenient to define a function $W(u)$ that satisfies

$$
\left(\frac{\delta W}{\delta u}\right)^{2} \equiv V(u)
$$

With this identification, the minimum energy $E$ in Eq. (9) is equal to a topological charge $Z$ defined by

$$
\begin{aligned}
Z & \equiv \int_{-\infty}^{\infty} d u\left(\frac{\delta W}{\delta u}\right) \\
& =W(u(\infty))-W(u(-\infty)) .
\end{aligned}
$$

The quantity $Z$ is topological in the sense that it depends purely on the boundary values of the field $u(x)$ at $x= \pm \infty$ and not on the field profile. Thus, the energy (or mass) of a dynamic kink (antikink) must be greater or equal (in the quasistatic limit) to $Z$. This is known as the BogomolnyPrasad-Sommerfield (BPS) bound [47-49]. Note that the two first-order field solutions Eq. (8) and the associated field energies are symmetric between a kink (+) and an antikink (-).

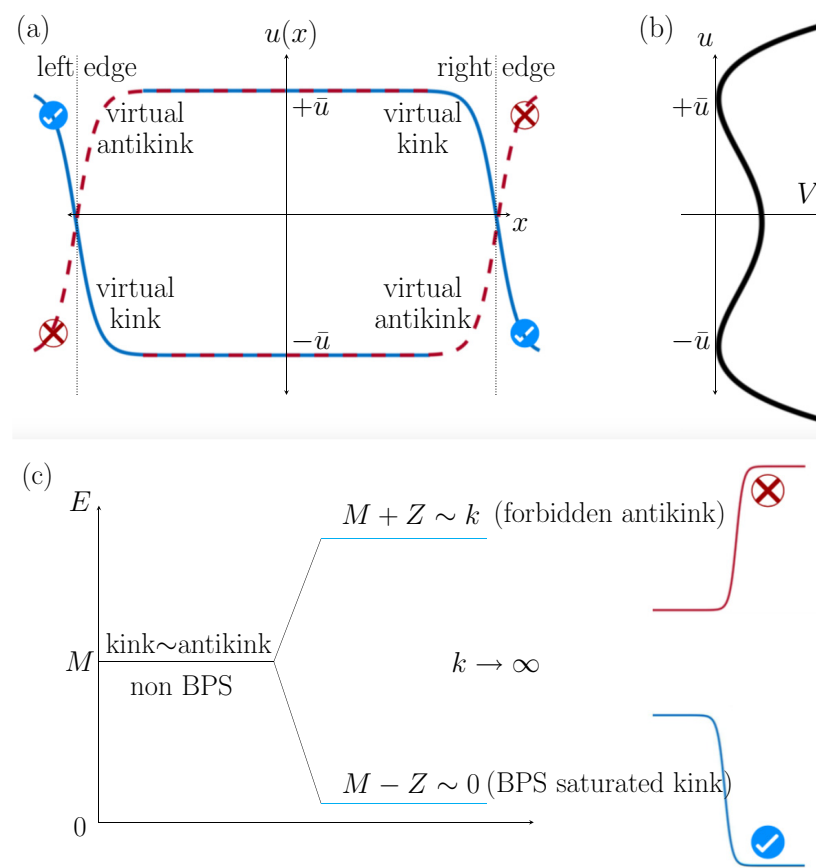

FIG. 2. (a) Illustrating the asymmetry between allowed kink states (solid curves) and forbidden antikink (dotted curves) states. A zero mode (virtual kink) localized at the right edge (uniform potential $+\bar{u}$ in the bulk) can propagate down the chain as a kink, and localize at the left edge (uniform potential $-\bar{u}$ in the bulk). (b) The double-well potential corresponding to the two kink states. (c) Energy diagram illustrating the kink-antikink asymmetry. As the spring constant $k \rightarrow \infty$, it takes an infinite energy to excite an antikink state.

In the case of Eq. (4), the kink solution (but not the antikink) makes the elastic energy vanish and there is no additional boundary term. The kink is said to saturate the BPS bound. At the same time, the apparent symmetry between a kink and antikink $(u \rightarrow-u)$ in Eq. (13) no longer exists, since the elastic energy in Eq. (4) only vanishes for the specific (static) configuration which satisfies Eq. (2), while it costs a finite energy for the other. Physically, a kink state in the bulk of the chain corresponds to right-leaning bars (green) on the left half of the chain and left-leaning bars, on the right side, with a nearly vertical bar in the middle of the domain wall; see Fig. 1. However, an antikink state will require left-leaning bars on the left side of the chain and right-leaning bars on the right side with a nearly vertical bar in the middle, and will thus require the orange connecting bars to be either of longer rest length or be stretched; see Refs. [16,17] for more details and pictures of a Lego realization. Since the kink profile is the finite-amplitude manifestation (in nonlinear theory) of the zero-energy edge mode (within linear theory), it can propagate down the chain without costing any energy. Thus, the asymmetry between the kink and antikink can be ultimately traced back to the existence of the localized edge mode under open boundary conditions.

We illustrate this crucial symmetry breaking in Fig. 2. In the top panel, we show the allowed and forbidden zero-mode configurations in the chain. According to the linearized theory, for a configuration with $\bar{u}>0(<0)$, the zero mode is initially "localized" at the right (left) edge. In Fig. 2, we refer to these 
edge-localized configurations as virtual kink and antikink, respectively, since in this state, only a part of their full profile is visible. However, when the nonlinear nature of the mechanical structure is taken into account, we find the zero mode develops into a real kink that can propagate down the chain and transition between the right and left localized states (or manifest as an intermediate state in the bulk of the chain; see also Fig. 1). At the same time, we never find a configuration which supports an antikink. This asymmetry is physically the result of a finite energy gap between the kink and antikink configurations; see Fig. 2 (bottom panel). In the next sections we demonstrate that in the supersymmetric version of the field theory, this asymmetry between kink and antikinks is related to a breaking of the supersymmetry.

Since the Euler-Lagrange equation of motion for Eq. (4) does not depend on the boundary term, the kink (antikink) solutions corresponding to the Hamiltonians in Eqs. (4) and (6) are identical. However, the boundary term does change the energy of kinks and antikinks. For both Eqs. (4) and (6), we obtain the nonlinear Klein-Gordon equation

$$
\frac{\partial^{2} u}{\partial t^{2}}-c^{2} \frac{\partial^{2} u}{\partial x^{2}}=-\frac{8 c^{2}}{a^{4}} u\left(u^{2}-\bar{u}^{2}\right) .
$$

The domain wall solution to Eq. (13) interpolates between left-leaning and right-leaning rotors, see Fig. 1, and carries with it the zero-energy mode as it propagates down the chain. This is reminiscent of how a domain wall facilitates electron transport in polyacetylene [63]. In polyacetylene, however, the kink is associated with bond distortions.

While we have taken the Ising-like kink as our main working example, we can apply the formalism developed in this paper also to other structures [16] like the helical realization in Fig. 3. In contrast to Fig. 1, this structure realizes the opposite limit $r \gg a$, and here we obtain the following nonlinear constraint equation

$$
\frac{d \theta}{d x}=-\frac{1}{r} \frac{\sin (\theta-\bar{\theta})}{\sin \bar{\theta}},
$$

instead of Eq. (2). The resulting dynamics is governed by the sine-Gordon equation [16]. In contrast with the Ising-like kink discussed so far, we now have spinning solitons: the rotating bars (shown in yellow in Fig. 3) undergo a rotation by $\pi$ and thus the dynamics is described in terms of the full angle $\theta(x)$ which the rotating bars make with the vertical. As seen in Fig. 3, in order to allow the rotating bars to rotate by $\pi$ without getting obstructed by the rigid base (contrast with Fig. 1), we need a staggered arrangement of bars, effectively embedding the structure in three dimensions. Rotating the zero-energy edge mode (shown in the far left end in Fig. 3) by $\pi$ shifts the zero mode to the adjacent rotor (along the $x$ axis), whose dynamics can effectively still be approximated by two copies of the one-dimensional sine-Gordon equation, one copy each for the rotors on odd and even sites respectively; see Ref. [16] for more details. For each copy of the sine-Gordon soliton, the supersymmetric extension discussed in the next section carries through by replacing $u(x) \rightarrow \theta(x)$ with

$$
\frac{\delta W(\theta)}{\delta \theta}= \pm \frac{1}{r} \frac{\sin (\theta \mp \bar{\theta})}{\sin \bar{\theta}}
$$

in Eqs. (10) and (4) for the odd (+) and even (-) sites.

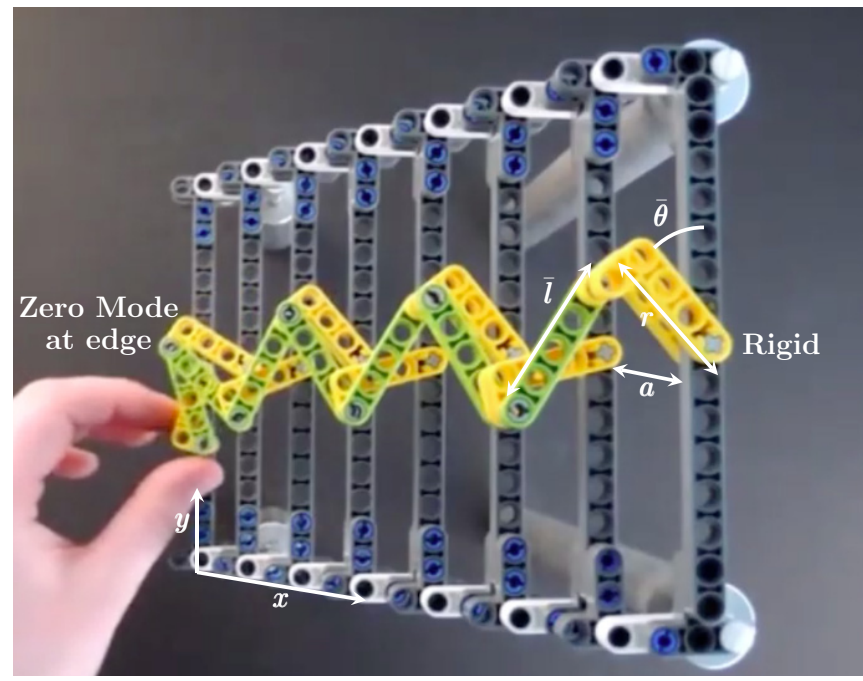

FIG. 3. A Lego realization of a structure where a zero-energy edge mode evolves into a sine-Gordon soliton that can start only from one of the two boundaries of the system [16]. During the propagation of this soliton, the rotating bars (yellow) undergo a rotation by $\pi$ and thus require a three-dimensional realization. The soliton is obtained as the solution of the constraint equation Eq. (14). Here, $\bar{\theta}$ is the equilibrium angle that the yellow rotors make with the vertical gray bars, $a$ is lattice spacing along the $x$ direction, $r$ is the length of the yellow bars, and the length of green connecting bars is $\bar{l}=\left(a^{2}+4 r^{2} \cos ^{2} \bar{\theta}\right)^{\frac{1}{2}}$; see Appendix B. These sine-Gordon kinks are obtained in the limit $r \gg a$. For a video, see the Supplemental Material [71].

\section{SUPERSYMMETRIC FIELD THEORY}

In order to further develop the connections between BPS kinks and supersymmetry and the emergence of fermionic variables, we apply Dirac's procedure to take the square root of the classical Hamiltonian in Eq. (4). Note that taking the square root of an equation of motion (where possible) is a useful method to simplify and reduce the order of an equation. For instance, the square root of the linear wave equation gives two first-order equations which are then used to construct the d'Alembert solutions. Likewise, the BPS method discussed in the last section allows us to obtain first-order equations directly from the Hamiltonian. Moreover, Eq. (12) indicates that for a kink configuration, the topological charge is defined via the square root of the potential term. Taking inspiration from these, we find that applying Dirac's square root procedure to a general Hamiltonian results in a dynamical charge if we allow anticommutating variables in the theory. As we discuss next, this charge corresponds to one of the conserved charges in a supersymmetric extension of the original classical theory. For a SUSY theory with two fermionic variables, we obtain as a by-product a second conserved charge, which defines a partner Hamiltonian to the original.

Note that the existence of a BPS kink [Eq. (3)] has allowed us to express the elastic term in Eq. (4) as a perfect square. Thus, we define a field-theoretic charge $Q_{1}$ of the form

$$
Q_{1}=\int d x\left[\pi \psi_{1}+c\left(\frac{\partial u}{\partial x}+\frac{\delta W}{\delta u}\right) \psi_{2}\right]
$$


where we introduce two real field variables $\psi_{1,2}(x, t)$ and a potential $W(u)$ which equals $W(u)=\frac{2}{a^{2}}\left(\bar{u}^{2} u-\frac{1}{3} u^{3}\right)$. We refer the reader unfamiliar with supersymmetry to the pedagogical treatment in Ref. [64] whose approach and notation we follow closely. In Appendix A, we show that in order for $Q_{1}^{2}=\mathcal{H}$, $\psi_{1,2}$ needs to satisfy the equal-time anticommutation relations:

$$
\left\{\psi_{a}(x, t), \bar{\psi}_{b}\left(x^{\prime}, t\right)\right\}=\left(\gamma^{0}\right)_{a b} \delta\left(x-x^{\prime}\right),
$$

where the index $a, b=\{1,2\}$ and $\bar{\psi}_{1}=i \psi_{2}$ and $\bar{\psi}_{2}=-i \psi_{1}$. Here and in the following, we make use of the following gamma matrices:

$$
\gamma^{0}=\sigma_{2}=\left(\begin{array}{cc}
0 & -i \\
i & 0
\end{array}\right), \quad \gamma^{1}=i \sigma_{3}=\left(\begin{array}{cc}
i & 0 \\
0 & -i
\end{array}\right) .
$$

Moreover, we can combine $\psi_{1,2} \equiv \psi_{1,2}(x, t)$ into a twocomponent Majorana field $\Psi \equiv \Psi(x, t)=\left(\begin{array}{l}\psi_{1} \\ \psi_{2}\end{array}\right)$ with its conjugate defined as $\bar{\Psi} \equiv \Psi^{\dagger} \gamma^{0}$. Note again that $\psi_{1,2}$ are real and, therefore, we refer to $\Psi$ as a Majorana field (the particle is the same as the antiparticle) [64].

Consider next the supersymmetric Lagrangian [52]

$$
\mathcal{L}_{s}=\mathcal{L}_{b}+\mathcal{L}_{f},
$$

where $\mathcal{L}_{b}$ is the bosonic part of the Lagrangian obtained from Eq. (4),

$$
\mathcal{L}_{b}=\frac{1}{2} \int d x\left[\left(\frac{\partial u}{\partial t}\right)^{2}-c^{2}\left(\frac{\partial u}{\partial x}-\frac{\delta W}{\delta u}\right)^{2}\right],
$$

while the Lagrangian $\mathcal{L}_{f}$ is expressed in terms of the Majorana field $\Psi$,

$$
\mathcal{L}_{f}=\frac{1}{2} \int d x i \bar{\Psi} \gamma^{\nu} \partial_{\nu} \Psi-\frac{\delta^{2} W}{\delta u^{2}} \bar{\Psi} \Psi,
$$

where $v=\{0,1\}$ denote time $(t)$ and space $(x)$ components, respectively; $\partial_{0} \rightarrow \partial_{t}, \partial_{1} \rightarrow c \frac{\partial}{\partial x}$, and $\bar{\Psi}=\Psi^{\dagger} \gamma^{0}$.

The action of Eq. (19) is invariant under supersymmetry transformations [65],

$$
\begin{gathered}
\delta u=i \epsilon_{2} \psi_{1}-i \epsilon_{1} \psi_{2}, \\
\delta \psi_{1}=-\dot{u} \epsilon_{2}+\epsilon_{1}\left(u^{\prime}-\frac{\delta W}{\delta u}\right), \\
\delta \psi_{2}=\dot{u} \epsilon_{1}-\epsilon_{2}\left(u^{\prime}+\frac{\delta W}{\delta u}\right),
\end{gathered}
$$

where $\epsilon_{1,2}$ are real anticommutating transformation parameters. The two conserved charges associated with this supersymmetry are $Q_{1}$ [Eq. (16)] and

$$
Q_{2}=\int d x\left[\pi \psi_{2}+c\left(\frac{\partial u}{\partial x}-\frac{\delta W}{\delta u}\right) \psi_{1}\right] .
$$

Note that $Q_{1}$ and $Q_{2}$ have different signs of the potential term, i.e., $\pm\left(\frac{\delta W}{\delta u}\right)$, respectively. Thus, they square to generate two different Hamiltonians

$$
\mathcal{H}_{1,2}=\frac{1}{2} \int d x \pi^{2}+c^{2}\left(\frac{\partial u}{\partial x}\right)^{2}+c^{2}\left(\frac{\delta W}{\delta u}\right)^{2} \pm 2 c^{2} \frac{\partial u}{\partial x} \frac{\delta W}{\delta u} .
$$

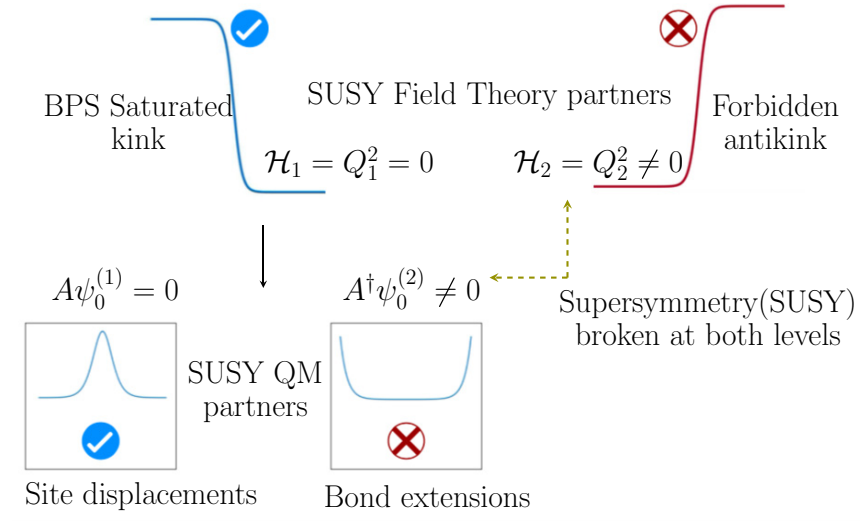

FIG. 4. Illustrating the half-broken supersymmetry at both the field-theoretic level (associated with the kink-antikink asymmetry) and in describing the fluctuations around the classical kink (associated with absence of bond stretching in mechanisms).

While $\mathcal{H}_{1}$ is the same as Eq. (4), $\mathcal{H}_{2}$ corresponds to a Hamiltonian generated from a constraint which yields an antikink profile, i.e., Eq. (3) with $u \rightarrow-u$. In supersymmetric theories $\mathcal{H}_{1,2}$ are called partner Hamiltonians; see Fig. 4. Note how the first three terms in Eqs. (26) reproduce the well known " $\phi$ " theory [e.g., in the SSH model and discussed in Eq. (6)] whose integral is the energy (or mass) of the kink or antikink solution which we denote by $M$ [64]. The last term in both the equations however is special since it is a total derivative whose integral only depends on the boundary conditions and hence it is a topological property of the mechanical structure; see Eq. (12). Thus, $Q_{1}^{2}=M+Z$ and $Q_{2}^{2}=M-Z$. Without the kinetic term (static solutions) in Eq. (4), $M \rightarrow M_{s}$ in which case $E=M_{s} \pm Z$ represents the elastic potential energy associated with the (anti)kink configurations. In the special case when either $M=Z$ or $M=-Z$, the elastic potential energy associated with one of the configurations is zero and this is the defining feature of a mechanism where the (BPS) bound $(M \geqslant Z)$ is saturated. In our case, the condition $M=-Z$ recovers the constraint equation (2) which we refer to as the kink profile. The antikink profile then has an elastic energy $E=2 Z$ and therefore the kink and antikink configurations are not symmetrical. In our framework, the kink and antikink are obtained from partner Hamiltonians defined through a supersymmetric extension of the classic theory.

Note also from Eq. (24) that the variation of the $\Psi$ field vanishes only if the BPS equation is satisfied for a static kink. In other words, the classical BPS solutions remain invariant under supersymmetry transformations, and thus supersymmetry is said to be half broken.

\section{SUPERSYMMETRIC QUANTUM MECHANICS}

In order to further clarify supersymmetry breaking (due to the BPS saturated kink) and the physical meaning of the Majorana field in the mechanical context, we next study small fluctuations around the kink. In the process, we reveal another supersymmetric structure inherent to the study of fluctuations, i.e., at the particle level, referred to as supersymmetric quantum mechanics [66]. 
As the first step, we linearize Eq. (13) around the kink solution $u_{s}(x, t)$ by expressing $u(x, t)=u_{s}(x, t)+\psi(x, t)$ and look for small distortions of the kink field in the form $\psi(x, t)=\psi_{n}^{(1)}(x) \exp \left(i \omega_{n} t\right)$. This in turn yields a Schrödingerlike equation for $\psi(x, t)$,

$$
\mathcal{H}_{1} \psi_{n}^{(1)}=\rho \omega_{n}^{2} \psi_{n}^{(1)},
$$

where $\mathcal{H}_{1}=c^{2}\left(-\frac{\partial^{2}}{\partial x^{2}}+U_{1}\right)$ is the second-order differential operator and we have defined the potential $U_{1}=\left[\left(\frac{d \tilde{V}}{d u}\right)^{2}+\right.$ $\left.\tilde{V} \frac{d^{2} \tilde{V}}{d u^{2}}\right]_{u=u_{s}(x)}$, with $\tilde{V}(u)=\frac{\delta W}{\delta u}$. Note that since $\mathcal{H}_{1}$ is a Hermitian differential operator, its eigenvectors $\psi_{n}^{(1)}$ constitute an orthogonal basis. In particular, the bound state solutions $\psi_{b}^{(1)}$ are real and satisfy the orthogonality condition $\psi_{b}^{(1)}(x) \psi_{b}^{(1)}\left(x^{\prime}\right) \propto \delta\left(x-x^{\prime}\right)$.

Next, by defining $w(x)=-\frac{\delta^{2} W}{\delta u^{2}}$, we can factorize $\mathcal{H}_{1}$ as a product of two first-order differential operators, $\mathcal{H}_{1}=A^{\dagger} A$, where

$$
A=-\frac{d}{d x}+w(x), \quad A^{\dagger}=\frac{d}{d x}+w(x) .
$$

Note that Eqs. (28) have a structure very similar to the static part of the charges $Q_{1,2}$, Eq. (16). Since $A^{\dagger} A \neq A A^{\dagger}$, we can define a Hamiltonian $\mathcal{H}_{2}=A A^{\dagger}$ with potential $U_{2}(x)=$ $\left[\left(\frac{d \tilde{V}}{d u}\right)^{2}-\tilde{V} \frac{d^{2} \tilde{V}}{d u^{2}}\right]$. Together $\mathcal{H}_{1,2}$ constitute a pair of quantum supersymmetric partner Hamiltonians; see also Fig. 4. In analogy with the notation used in quantum mechanics, we label operator $A$ as a lowering operator and $A^{\dagger}$ as a raising operator and discuss next their physical meaning for our mechanical system.

Physically, the bound states of $\mathcal{H}_{1}(x)$ are the site displacement eigenfunctions. Applying the lowering operator once, we obtain the corresponding bond extensions, $\psi_{n}^{(2)}(x)=$ $A \psi_{n}^{(1)}$, or equivalently the tensions $k \psi_{n}^{(2)}(x)$ that would be measured in an elastic structure. The operator $A$ is thus a continuum version of the discrete rigidity matrix $R$; see Appendix $\mathrm{B}$ for further details. Alternately, the bond extensions $\psi_{n}^{(2)}(x)$ are obtained directly as the bound states of $U_{2}$, the potential associated with the partner Hamiltonian $\mathcal{H}_{2}$. The Hamiltonians $\mathcal{H}_{1}, \mathcal{H}_{2}$ are said to be partners because once the eigenvalues (eigenfunctions) of $\mathcal{H}_{1}$ are known, the corresponding ones for $\mathcal{H}_{2}$ can be easily obtained (except for the zero-energy eigenvalue that we assume to be part of $\mathcal{H}_{1}$ as discussed below). Thus for example, if $\mathcal{H}_{1} \psi_{n}^{(1)}=E_{n}^{(1)} \psi_{n}^{(1)}$, then $\mathcal{H}_{2}\left(A \psi_{n}^{(1)}\right)=A A^{\dagger} A \psi_{n}^{(1)}=E_{n}^{(1)}\left(A \psi_{n}^{(1)}\right)$. Each eigenfunction in $\mathcal{H}_{1}$ has a partner in the spectrum of $\mathcal{H}_{2}$ except for the ground state defined via $\mathcal{H}_{1} \psi_{0}=A^{\dagger} A \psi_{0}=0$. The sitedisplacement field is obtained from the bond-stretching field by applying the raising operator, i.e., $\psi_{n}^{(1)}(x)=A^{\dagger} \psi_{n}^{(2)}$.

The zero-energy bound states which are obtained by solving the pair of Eqs. (28) with $w(x)=\frac{4 u_{s}}{a^{2}}=\frac{-4 \bar{u}}{a^{2}} \tanh \left(\frac{2 \bar{u} x}{a^{2}}\right)$ are however special. Solving for $A \psi_{0}^{(1)}=0$ (equivalently $\mathcal{H}_{1} \psi_{0}^{(1)}=0$ ), we obtain

$$
\psi_{0}^{(1)}(x) \propto \operatorname{sech}^{2}\left(\frac{2 \bar{u}}{a^{2}} x\right)
$$

while from $A^{\dagger} \psi_{0}^{(2)}=0$ (equivalently $\mathcal{H}_{2} \psi_{0}^{(2)}=0$ ), we obtain

$$
\psi_{0}^{(2)}(x) \propto \cosh ^{2}\left(\frac{2 \bar{u}}{a^{2}} x\right) .
$$

The proportionality constants for Eqs. (29) and (30) are obtained by requiring the solutions to be normalizable. While Eq. (29) is always normalizable, Eq. (30) grows exponentially with the system size and is thus physically not observable in a large sample (it is localized to the sample edges). In the quantum regime, this "unpairing" of the zero modes results in the curious phenomena of fractional fermion number $[63,67,68]$. However, this is to be contrasted with our mechanical system where even if the system size remains small, Eq. (30) is not physically realizable when we consider mechanisms, i.e., when the spring constant $k \rightarrow \infty$. This is because $A^{\dagger}$ corresponds to bond extensions which are forbidden in the mechanism limit and thus the only permissible solution to Eq. (30) is

$$
\psi_{0}^{(2)}(x)=0 .
$$

On the other hand, the orthogonality of the modes leads to a normalization factor $C=\left(\frac{3 a^{2}}{8 \bar{u}^{3}}\right)^{\frac{1}{2}}$ for $\psi_{0}^{(1)}$ via the integral

$$
C^{2}\left(\frac{2 \bar{u}}{a^{2}}\right)^{2} \int_{-\infty}^{\infty} d x \operatorname{sech}^{4}\left(\frac{2 \bar{u}}{a^{2}} x\right)=1 .
$$

We thus see that only one of $\mathcal{H}_{1} \psi_{0}^{(1)}$ or $\mathcal{H}_{2} \psi_{0}^{(2)}$ can be zero, reminiscent of the asymmetry and hence broken symmetry between the field-theoretic charges and their corresponding partner Hamiltonians Eq. (26). In mechanisms, the source of both of these asymmetries is the absence of bond stretching and, hence, forbidden antikink $\mathcal{Q}_{2} \neq 0$ and forbidden zero mode $A^{\dagger} \psi_{0}^{(2)} \neq 0$. This is illustrated in Fig. 4, where we show the symmetry breaking both between a kink and antikink (SUSY field theory), and between the zero modes and the states of self-stress around the (anti)kink solution (SUSY quantum mechanics).

We now define the index $v$ of the operator $A$ as the difference in the dimension of the kernel of $A$ and $A^{\dagger}$. Using the identities $\operatorname{ker} A=\operatorname{ker} A^{\dagger} A$ and $\operatorname{ker} A^{\dagger}=\operatorname{ker} A A^{\dagger}$, we obtain the Witten index [70]

$$
v=\operatorname{dim} \operatorname{ker} \mathcal{H}_{1}-\operatorname{dim} \operatorname{ker} \mathcal{H}_{2} .
$$

The mechanical interpretation of this field-theoretic statement comes from realizing that $\mathcal{H}_{1}=A^{\dagger} A$ is a real-space continuum generalization of the discrete dynamical matrix $R^{T} R$. Hence the dimension of its kernel gives the number of zero-energy displacement modes whereas $\mathcal{H}_{2}=A A^{\dagger}$ (corresponding to $R R^{T}$ ) gives the states of self-stress. Thus the Witten index (generally defined for supersymmetric theories) reduces to the index obtained in Eq. (1) from the Maxwell count and derived within topological band theory in Ref. [12]. For the chain mechanism $v=1$, there is only one normalizable zero-energy eigenstate $\psi_{0}^{(1)}$ that we associate with $\mathcal{H}_{1}$.

Combining $\mathcal{H}_{1}, \mathcal{H}_{2}$, we get the matrix $\mathcal{H}$ that together with the operators $\mathcal{Q}$ and $\mathcal{Q}^{\dagger}$ given by

$$
\mathcal{H}=\left(\begin{array}{cc}
\mathcal{H}_{1} & 0 \\
0 & \mathcal{H}_{2}
\end{array}\right), \quad \mathcal{Q}=\left(\begin{array}{cc}
0 & 0 \\
A & 0
\end{array}\right), \quad \mathcal{Q}^{\dagger}=\left(\begin{array}{cc}
0 & A^{\dagger} \\
0 & 0
\end{array}\right)
$$




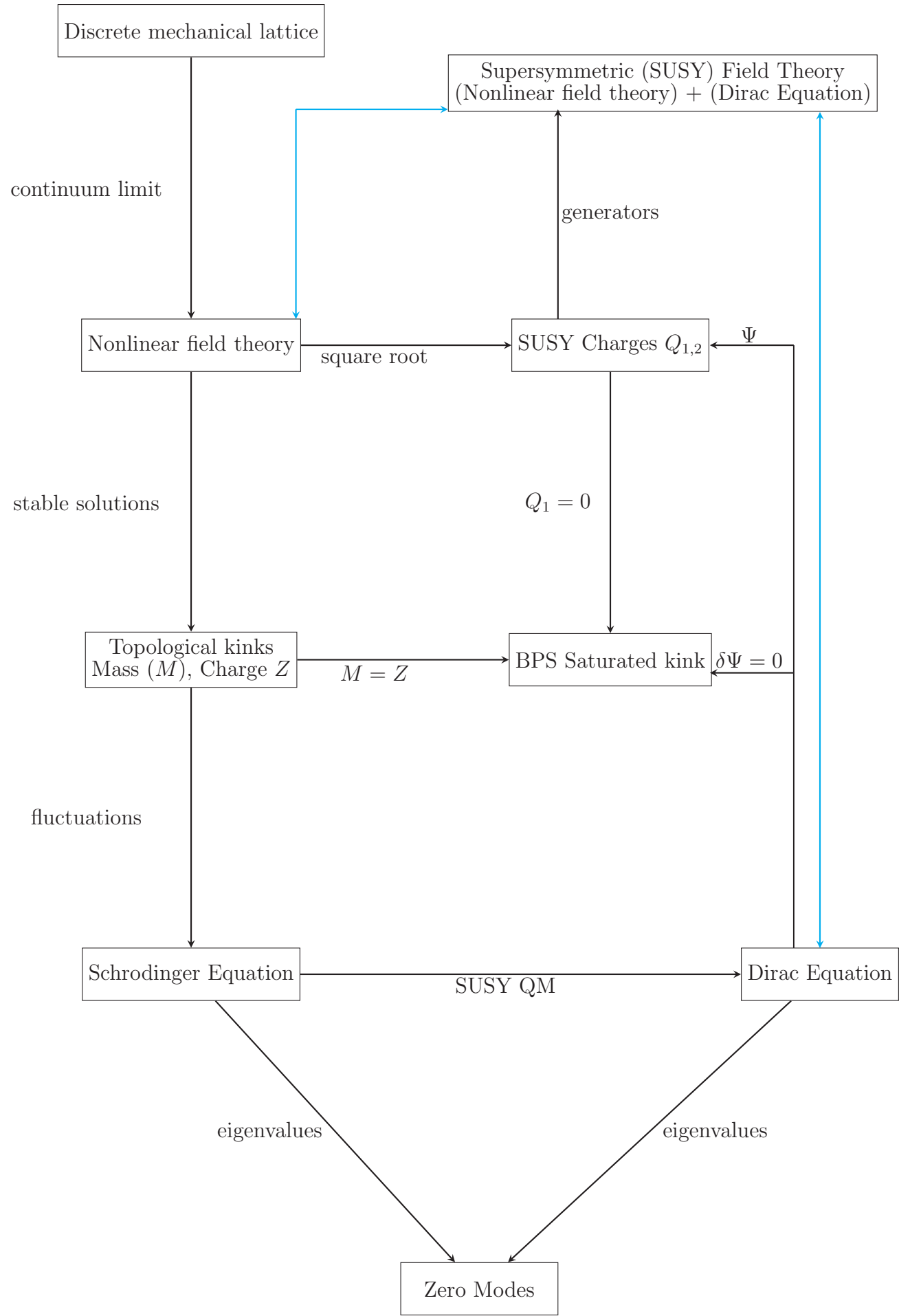

FIG. 5. A map of the theoretical framework used in our work. Beginning with a discrete lattice shown in Fig. 1, we take the continuum limit to obtain Eq. (4) with kink solutions. The study of fluctuations around the kink leads to the Schrödinger equation Eq. (27), which can be decomposed into two first-order operators, referred to as charges in supersymmetric quantum mechanics. The first-order operators are in turn identified with the Dirac equation Eq. (35). Equivalently, the square root of the nonlinear field theory in Eq. (4) leads to charge Eq. (16) provided we allow Majorana fermions in our theory. The charge can be identified with conserved charges of the SUSY field-theoretic Lagrangian Eq. (19).

satisfies the superalgebra, $[\mathcal{H}, \mathcal{Q}]=\left[\mathcal{H}, \mathcal{Q}^{\dagger}\right]=0$, $\left\{\mathcal{Q}, \mathcal{Q}^{\dagger}\right\}=\mathcal{H}$, and $\{\mathcal{Q}, \mathcal{Q}\}=\left\{\mathcal{Q}^{\dagger}, \mathcal{Q}^{\dagger}\right\}=0 \quad$ [51,70]. The two-component field $\Psi_{n}$, formed by combining $\psi_{n}^{(1)}$ and $\psi_{n}^{(2)}$, can itself be viewed as a "fermion" field, as evidenced by the anticommuting algebra of the $\mathcal{Q}$ and $\mathcal{Q}^{\dagger}$ operators. 


\section{MECHANICAL MAJORANA MODES}

As hinted by the fermionic character of $\Psi$, the same results we have derived in the previous section can be compactly obtained from the Dirac Lagrangian $\mathcal{L}_{f}$, Eq. (19), with inhomogeneous mass $w(x)=-\frac{\delta^{2} W(u)}{\delta u^{2}}$ :

$$
\mathcal{L}=i \bar{\Psi} \gamma^{\mu} \partial_{\mu} \Psi+\bar{\Psi} \Psi w(x) .
$$

The corresponding Euler-Lagrange equation of motion,

$$
i \gamma^{\mu} \partial_{\mu} \Psi+w(x) \Psi=0,
$$

is a Dirac equation where the constant mass term is replaced by the inhomogeneous field $-w(x)[67,68]$. The classical Majorana zero mode minimizes its energy by localizing where $w(x)=-\frac{\delta}{2} W(u)_{\delta u^{2}}$ is vanishingly small, i.e., in the middle of the domain wall for the chain in Fig. 1 or at the core of topological defects in more complex 2D structures $[18,69]$.

We now seek solutions of Eq. (36) of the form $\Psi(x, t)=$ $\Psi_{n}(x) \exp \left(i \omega_{n} t\right)$ and obtain

$$
-\gamma^{0} \omega_{n} \Psi_{n}(x)+i \gamma^{1} c \frac{d \Psi_{n}}{d x}+w(x) \Psi_{n}(x)=0,
$$

where the Majorana field $\Psi_{n}(x)$ and the corresponding gamma matrices $\left\{\gamma^{0}, \gamma^{1}\right\}$ (Majorana basis) are

$$
\Psi_{n}(x)=\left(\begin{array}{c}
\psi_{n}^{(1)} \\
\psi_{n}^{(2)}
\end{array}\right), \quad \gamma^{0}=\left(\begin{array}{cc}
0 & -i \\
i & 0
\end{array}\right), \quad \gamma^{1}=\left(\begin{array}{cc}
i & 0 \\
0 & -i
\end{array}\right) \text {. }
$$

With the above choices, the two components of the Dirac equation assume the form

$$
\begin{aligned}
& A \psi_{n}^{(1)}(x)=-i \omega_{n} \psi_{n}^{(2)}(x), \\
& A^{\dagger} \psi_{n}^{(2)}(x)=i \omega_{n} \psi_{n}^{(1)}(x),
\end{aligned}
$$

from which we recover the same eigenvalue problem $A^{\dagger} A \psi_{n}^{(1)}=\omega_{n}^{2} \psi_{n}^{(1)}$ derived in the previous section. The crucial point is that the operators $A$ and $A^{\dagger}$ are exactly the ones derived in Eq. (28). As a result, $\left\{\psi_{n}^{(1)}, \psi_{n}^{(2)}\right\}$ are the eigenstates of the doubled Hamiltonian $\mathcal{H}$ in Eq. (34). Correspondingly, the zero modes of $A, A^{\dagger}$ are given by Eqs. (29) and (31), respectively.

\section{CONCLUSION}

We summarize the theoretical framework and the connections between different ideas used in our work in Fig. 5. Beginning with a discrete lattice shown in Fig. 1, we take the continuum limit to obtain the nonlinear field theory [Eq. (4)] with a BPS saturated kink that comes from enforcing the mechanical constraint Eq. (2). We then generalize Dirac's procedure to take the square root of the nonlinear field theory and obtain fermionic (Majorana) charges, which in turn correspond to symmetries of the Witten-Olive supersymmetric theory Eq. (19). In order to understand the physical meaning of the fermionic variables, we then study fluctuations around the kink solution and obtain a Schrödinger-like equation
Eq. (27), which can be viewed as a product of two first-order Dirac-like operators. These Dirac-like operators physically describe site displacements and bond extensions of the lattice around stable configurations, which could either be the uniform state (considered in Ref. [12]) or the inhomogeneous kink state, where nonlinearities are fully taken into account. Further, we show that the Dirac operators can equivalently be obtained as the equation of motion from the fermionic part of the supersymmetric Lagrangian Eq. (19).

Aside from the examples explicitly considered here, our approach when run in reverse could provide a systematic strategy to model nontrivial topological mechanical structures starting from well-classified supersymmetric field theories of which the Witten-Olive theory is only the simplest example [64].

\section{ACKNOWLEDGMENTS}

N.U. and V.V. were supported by the Complex Dynamics and Systems Program of the Army Research Office under Grant No. W911NF-19-1-0268. V.V. acknowledges support from the Simons Foundation and by the University of Chicago Materials Research Science and Engineering Center, which is funded by the National Science Foundation under Award No. DMR-2011854. We acknowledge financial support from FOM, NWO, and the Delta Institute for Theoretical Physics. We thank A. Achúcarro, R. D. Kamien, R. J. Slager, Y. Kafri, E. Cobanera, C. Beenakker, A. Akhmerov, J. Paulose, A. M. Turner, C. Scheibner, and M. Fruchart for stimulating conversations. We thank A. Meeussen for creating movies of the prototypes. We thank ESPCI, Paris, for our stay there during the summer of 2014, where part of this work was conceived.

\section{APPENDIX A: SUPERSYMMETRY GENERATORS} form

We chose our basis such that the $\gamma$ matrices assume the

$$
\gamma^{0}=\left(\begin{array}{cc}
0 & -i \\
i & 0
\end{array}\right)
$$

and

$$
\gamma^{1}=\left(\begin{array}{cc}
i & 0 \\
0 & -i
\end{array}\right)
$$

The supercharges $Q_{1,2}$ are then

$$
\begin{aligned}
Q_{1} & =\int d x\left[\frac{\partial u}{\partial t} \psi_{1}+c\left(\frac{\partial u}{\partial x}+\frac{\delta W}{\delta u}\right) \psi_{2}\right] \\
Q_{2} & =\int d x\left[\frac{\partial u}{\partial t} \psi_{2}+c\left(\frac{\partial u}{\partial x}-\frac{\delta W}{\delta u}\right) \psi_{1}\right] .
\end{aligned}
$$

The charges generate supertransformations of the fields,

$$
\left[Q_{\alpha}, u\right]=-i \psi_{\alpha},\left\{Q_{\alpha}, \bar{\psi}_{\beta}\right\}=\left(\gamma^{v} \partial_{v}\right)_{\alpha \beta} u+i \frac{\partial W}{\partial u} \delta_{\alpha \beta},
$$

where $\alpha, \beta=\{1,2\}$. 
Computing $Q_{1}^{2}$, we find

$$
\begin{aligned}
Q_{1}^{2}= & \iint d x d x^{\prime}\left[\frac{\partial u(x)}{\partial t} \frac{\partial u\left(x^{\prime}\right)}{\partial t} \psi_{1}(x) \psi_{1}\left(x^{\prime}\right)\right]+c\left(\frac{\partial u(x)}{\partial t}\left[\frac{\partial u\left(x^{\prime}\right)}{\partial x^{\prime}}+\frac{\delta W\left(u\left(x^{\prime}\right)\right)}{\partial u}\right] \psi_{1}(x) \psi_{2}\left(x^{\prime}\right)\right) \\
& +c\left[\frac{\partial u(x)}{\partial x}+\frac{\delta W(u(x))}{\partial u}\right] \frac{\partial u\left(x^{\prime}\right)}{\partial t} \psi_{2}(x) \psi_{1}\left(x^{\prime}\right)+c^{2}\left[\frac{\partial u(x)}{\partial x}+\frac{\delta W(u(x))}{\partial u}\right]\left[\frac{\partial u\left(x^{\prime}\right)}{\partial x^{\prime}}+\frac{\delta W\left(u\left(x^{\prime}\right)\right)}{\partial u}\right] \psi_{2}(x) \psi_{2}\left(x^{\prime}\right) .
\end{aligned}
$$

By interchanging the order of integrals involving variables $x, x^{\prime}$, we can express Eq. (A6) in the form

$$
\begin{aligned}
2 Q_{1}^{2}= & \iint d x d x^{\prime}\left(\frac{\partial u(x)}{\partial t} \frac{\partial u\left(x^{\prime}\right)}{\partial t}\left\{\psi_{1}(x), \psi_{1}\left(x^{\prime}\right)\right\}\right)+c\left(\frac{\partial u(x)}{\partial t}\left[\frac{\partial u\left(x^{\prime}\right)}{\partial x^{\prime}}+\frac{\delta W\left(u\left(x^{\prime}\right)\right)}{\partial u}\right]\left\{\psi_{1}(x), \psi_{2}\left(x^{\prime}\right)\right\}\right) \\
& +c\left[\frac{\partial u(x)}{\partial x}+\frac{\delta W(u(x))}{\partial u}\right] \frac{\partial u\left(x^{\prime}\right)}{\partial t}\left\{\psi_{2}(x), \psi_{1}\left(x^{\prime}\right)\right\}+c^{2}\left[\frac{\partial u(x)}{\partial x}+\frac{\delta W(u(x))}{\partial u}\right]\left[\frac{\partial u\left(x^{\prime}\right)}{\partial x^{\prime}}+\frac{\delta W\left(u\left(x^{\prime}\right)\right)}{\partial u}\right]\left\{\psi_{2}(x), \psi_{2}\left(x^{\prime}\right)\right\} .
\end{aligned}
$$

We next make use of the anticommutation relation [64]

$$
\left\{\psi_{\alpha}(x, t), \bar{\psi}_{\beta}\left(x^{\prime}, t\right)\right\}=\left(\gamma^{0}\right)_{\alpha \beta} \delta\left(x-x^{\prime}\right),
$$

where $\bar{\psi}=\psi^{\dagger} \gamma^{0}$. Therefore, $\bar{\psi}_{1}=i \psi_{2}$ and $\bar{\psi}_{2}=-i \psi_{1}$. Thus,

$$
\begin{aligned}
& \left\{\psi_{1}(x, t), \psi_{1}\left(x^{\prime}, t\right)\right\}=i\left\{\psi_{1}(x, t), \bar{\psi}_{2}\left(x^{\prime}, t\right)\right\}=\delta\left(x-x^{\prime}\right), \\
& \left\{\psi_{2}(x, t), \psi_{2}\left(x^{\prime}, t\right)\right\}=-i\left\{\psi_{2}(x, t), \bar{\psi}_{1}\left(x^{\prime}, t\right)\right\}=\delta\left(x-x^{\prime}\right),
\end{aligned}
$$

with the rest being 0 .

Substituting Eqs. (A9) into Eq. (A7), we obtain Eq. (26) in the main text.

\section{APPENDIX B: RIGIDITY MATRIX}

The Lagrangian for a chain with $n$ rotors is

$$
\mathcal{L}=\sum_{n} \frac{1}{2} I\left(\frac{d \theta_{n}}{d t}\right)^{2}-\frac{1}{2} K\left(l_{n, n+1}-\bar{l}\right)^{2},
$$

where $I=m r^{2}$ is the moment of inertia of a rotor of length $r$ and mass $m . K$ is the bare spring constant of the bar joining two adjacent rotors at sites $n, n+1$ whose squared length can be given by the expression [12]

$$
l_{n, n+1}^{2}=a^{2}+2 r^{2} \cos \left(\theta_{n}+\theta_{n+1}\right)+2 \operatorname{ar}\left[\sin \theta_{n}-\sin \theta_{n+1}\right],
$$

where $\theta$ is the angle with respect to the vertical measured positive in the clockwise direction and $a$ is the lattice spacing. In the uniform state, $\left|\theta_{n}\right|=\left|\theta_{n+1}\right|=\bar{\theta}$ and $l_{n, n+1}=\bar{l}=$ $\sqrt{a^{2}+4 r^{2} \cos ^{2} \bar{\theta}}$ for all $n$. The Euler-Lagrange equation of motion for the $n$th rotor is

$$
I \ddot{\theta}_{n}=-K\left(l_{n, n+1}-\bar{l}\right) \frac{\partial l_{n, n+1}}{\partial \theta_{n}}-K\left(l_{n-1, n}-\bar{l}\right) \frac{\partial l_{n-1, n}}{\partial \theta_{n}} .
$$

Shifting the index $n$ by one unit, we obtain

$$
I \ddot{\theta}_{n-1}=-K\left(l_{n-1, n}-\bar{l}\right) \frac{\partial l_{n-1, n}}{\partial \theta_{n-1}}-K\left(l_{n-2, n-1}-\bar{l}\right) \frac{\partial l_{n-2, n-1}}{\partial \theta_{n-1}} .
$$

Within linear approximation in the angular displacements (i.e., $\theta_{n}=\bar{\theta}-\delta_{n}$ ), Eq. (B1) can be approximated as

$$
l_{n, n+1}^{2}=\bar{l}^{2}+2 r^{2} \sin 2 \bar{\theta}\left(\delta_{n}+\delta_{n-1}\right)-2 \operatorname{arcos} \bar{\theta}\left(\delta_{n}-\delta_{n+1}\right),
$$

from where the infinitesimal change in the length of the spring $l_{n, n+1}^{2}=\left(\bar{l}+\delta l_{n, n+1}\right)^{2} \approx \bar{l}^{2}+2 \bar{l} \delta l_{n, n+1}$ can be expressed in the form

$$
\delta l_{n, n+1}=q_{+} \delta_{n}+q_{-} \delta_{n+1},
$$

where $q_{ \pm}=\frac{r \cos \bar{\theta}(2 r \sin \bar{\theta} \pm a)}{\bar{l}}$.

The linearized equation of motion Eqs. (B2) and (B3) is then

$$
\begin{aligned}
-\ddot{\delta}_{n} & =\left(q_{+}^{2}+q_{-}^{2}\right) \delta_{n}+q_{+} q_{-}\left(\delta_{n+1}+\delta_{n-1}\right), \\
-\ddot{\delta}_{n-1} & =\left(q_{+}^{2}+q_{-}^{2}\right) \delta_{n-1}+q_{+} q_{-}\left(\delta_{n}+\delta_{n-2}\right) .
\end{aligned}
$$

Expressing Eqs. (B5) in terms of normal coordinates with the choice $\delta_{n-2}=c_{1} e^{i[k(n-2) a-\omega t]}, \delta_{n-1}=c_{2} e^{i(k n a-\omega t)}, \delta_{n}=$ $c_{1} e^{i(k n a-\omega t)}, \delta_{n+1}=c_{2} e^{i[k(n+2) a-\omega t]}$, where $k$ is the wave number, we obtain a matrix equation:

$$
\omega^{2}\left(\begin{array}{l}
c_{1} \\
c_{2}
\end{array}\right)=D(k)\left(\begin{array}{l}
c_{1} \\
c_{2}
\end{array}\right),
$$

where $D(k)$ is the $2 \times 2$ dynamical matrix $[12,16]$

$$
D(k)=\left(\begin{array}{cc}
q_{+}^{2}+q_{-}^{2} & \left(1+e^{2 i k a}\right) q_{+} q_{-} \\
\left(1+e^{-2 i k a}\right) q_{+} q_{-} & q_{+}^{2}+q_{-}^{2}
\end{array}\right) .
$$

In order to take the square root of the dynamical matrix, we seek a matrix $R(k)$, such that $R^{\dagger}(k) R(k)=D(k)$ [12]. Consider a general $2 \times 2$ complex matrix

$$
R(k)=\left(\begin{array}{cc}
w & x \\
y & z
\end{array}\right),
$$

where $w, x, y, z$ are as yet undetermined complex functions of $k$. In order to find these functions, we first evaluate $R^{\dagger}(k)$ and then compare the product of $R^{\dagger} R$ to $D(k)$. This gives us the following three relations:

$$
\begin{gathered}
|w|^{2}+|y|^{2}=q_{+}^{2}+q_{-}^{2}, \\
|x|^{2}+|z|^{2}=q_{+}^{2}+q_{-}^{2}, \\
w^{*} x+y^{*} z=q_{+} q_{-}\left(1+e^{2 i k a}\right) .
\end{gathered}
$$

A possible choice that satisfies the first constraint Eq. (B8) is $w=q_{+}$and $y=q_{-}$. Next, if we chose $x=q_{-} e^{i 0}$ and $z=$ $q_{+} e^{2 i k a}$, we can satisfy both the constraint equations Eq. (B9) 
and Eq. (B10). Thus, a possible choice for $R(k)$ is

$$
R(k)=\left(\begin{array}{cc}
q_{+} & q_{-} \\
q_{-} & q_{+} e^{2 i k a}
\end{array}\right) .
$$

This is the rigidity matrix in Fourier space.

Physically, the rigidity matrix relates site displacements $u$ to bond elongations $\delta l$ in real space. To identify the first-order differential operator $A$ in Eq. (28) with the rigidity matrix in real space, consider again a pair of adjacent sites $n, n+1$ in Fig. 1. The bond extensions are $\delta l_{n, n+1}=\frac{2}{\bar{l}}\left[\frac{a}{2} r \cos \bar{\theta}\left(\delta \theta_{n}-\right.\right.$ $\left.\left.\delta \theta_{n+1}\right)+r^{2} \sin \bar{\theta} \cos \bar{\theta}\left(\delta \theta_{n}+\delta \theta_{n+1}\right)\right]$, where $\delta \theta_{n}, \delta \theta_{n+1}$ are small angular displacements around the homogeneous background $\theta=\bar{\theta}$.

A continuum limit of the distortion field $r \cos \bar{\theta} \delta \theta_{n} \rightarrow$ $u(x), r \cos \bar{\theta}\left(\delta \theta_{n}-\delta \theta_{n+1}\right) \rightarrow-\partial_{x} u$ reproduces the operator $A$ in Eq. (28) for the special case of a constant potential $w(x)=-\left(\frac{d V}{d u}\right)_{u=\bar{u}}=2 r \sin \bar{\theta}=2 \bar{u}$. However, when we expand around the soliton field as in Eq. (27), we obtain bond extensions over an inhomogeneous zero-energy state. Thus in general, the operator $A$ is a continuum limit of the real-space rigidity matrix around a specific solution of the nonlinear field theory and can be explicitly determined using Eqs. (28) and the superpotential $w(x)$.
[1] M. F. Thorpe, Continuous deformations in random networks, J. Non-Cryst. Solids 57, 355 (1983).

[2] K. Sun, A. Souslov, X. Mao, and T. C. Lubensky, Surface phonons, elastic response, and conformal invariance in twisted kagome lattices, Proc. Natl. Acad. Sci. USA 109, 12369 (2012).

[3] Z. Y. Wei, Z. V. Guo, L. Dudte, H. Y. Liang, and L. Mahadevan, Geometric Mechanics of Periodic Pleated Origami, Phys. Rev. Lett. 110, 215501 (2013).

[4] M. A. Dias, L. Dudte, L. Mahadevan, and C. D. Santangelo, Geometric Mechanics of Curved Crease Origami, Phys. Rev. Lett. 109, 114301 (2012).

[5] M. Schenk and S. Guest, Geometry of Miura-folded metamaterials, Proc. Natl. Acad. Sci. USA 110, 3276 (2013).

[6] F. G. Woodhouse, H. Ronellenfitsch, and J. Dunkel, Autonomous Actuation of Zero Modes in Mechanical Networks Far from Equilibrium, Phys. Rev. Lett. 121, 178001 (2018).

[7] M. Fruchart, S. Y. Jeon, K. Hur, V. Cheianov, U. Wiesner, and V. Vitelli, Soft self-assembly of Weyl materials for light and sound, Proc. Natl. Acad. Sci. USA 115, E3655 (2018).

[8] D. Z. Rocklin, V. Vitelli, and X. Mao, Folding mechanisms at finite temperature, arXiv:1802.02704.

[9] C. Scheibner, W. T. M. Irvine, and V. Vitelli, Non-Hermitian Band Topology and Skin Modes in Active Elastic Media, Phys. Rev. Lett. 125, 118001 (2020).

[10] P. A. M. Dirac, Lectures on Quantum Mechanics (Dover, New York, 2001).

[11] L. D. Faddeev and V. N. Popov, Feynman Diagrams for the Yang-Mills Field (Dover, New York, 2001).

[12] C. L. Kane and T. C. Lubensky, Topological boundary modes in isostatic lattices, Nat. Phys. 10, 39 (2014).

[13] V. Vitelli, Topological soft matter: Kagome lattices with a twist, Proc. Natl. Acad. Sci. USA 109, 12266 (2012).

[14] K. Bertoldi, V. Vitelli, J. Christensen, and M. V. Hecke, Flexible mechanical metamaterials, Nat. Rev. Mater. 2, 17066 (2017).

[15] S. D. Huber, Topological mechanics, Nat. Phys. 12, 621 (2016).

[16] B. G. Chen, N. Upadhyaya, and V. Vitelli, Nonlinear conduction via solitons in a topological mechanical insulator, Proc. Natl. Acad. Sci. USA 111, 13004 (2014).

[17] Y. Zhou, B. G. Chen, N. Upadhyaya, and V. Vitelli, Kinkantikink asymmetry and impurity interactions in topological mechanical chains, Phys. Rev. E 95, 022202 (2017).

[18] J. Paulose, B. G. Chen, and V. Vitelli, Topological modes bound to dislocations in mechanical metamaterials, Nat. Phys. 11, 153 (2015).
[19] J. Paulose, A. S. Meeussen, and V. Vitelli, Selective buckling via states of self-stress in topological metamaterials, Proc. Natl. Acad. Sci. USA 112, 7639 (2015).

[20] Y. Ran, Y. Zhang, and A. Vishwanath, One-dimensional topologically protected modes in topological insulators with lattice dislocations, Nat. Phys. 5, 298 (2009).

[21] J. C. Y. Teo and C. L. Kane, Topological defects and gapless modes in insulators and superconductors, Phys. Rev. B 82, 115120 (2010).

[22] V. Juričić, A. Mesaros, R.-J. Slager, and J. Zaanen, Universal Probes of Two-Dimensional Topological Insulators: Dislocation and $\pi$ Flux, Phys. Rev. Lett. 108, 106403 (2012).

[23] M. Z. Hasan and C. L. Kane, Colloquium: Topological insulators, Rev. Mod. Phys. 82, 3045 (2010).

[24] S. H. Mousavi, A. B. Khanikaev, and Z. Wang, Topologically protected elastic waves in phononic metamaterials, Nat. Commun. 6, 8682 (2015).

[25] R. Süsstrunk and D. H. Sebastian, Classification of topological phonons in linear mechanical metamaterials, Proc. Natl. Acad. Sci. USA 113, E4767 (2016).

[26] A. P. Deymier, K. Runge, N. Swinteck, and K. Muralidharan, Torsional topology and fermion-like behavior of elastic waves in phononic structures, C. R. Mec. 343, 700 (2015).

[27] R. Bi and Z. Wang, Unidirectional transport in electronic and photonic Weyl materials by Dirac mass engineering, Phys. Rev. B 92, 241109 (2015).

[28] V. Peano, C. Brendel, M. Schmidt, and F. Marquardt, Topological Phases of Sound and Light, Phys. Rev. X 5, 031011 (2015).

[29] Y. T. Wang, P. Luan, and S. Zhang, Coriolis force induced topological order for classical mechanical vibrations, New J. Phys. 17, 073031 (2015).

[30] P. Wang, L. Lu, and K. Bertoldi, Topological Phononic Crystals with One-Way Elastic Edge Waves, Phys. Rev. Lett. 115, 104302 (2015).

[31] H. C. Po, Y. Bahri, and A. Vishwanath, Phonon analog of topological nodal semimetals, Phys. Rev. B 93, 205158 (2014).

[32] A. S. Meeussen, J. Paulose, and V. Vitelli, Geared Topological Metamaterials with Tunable Mechanical Stability, Phys. Rev. X 6, 041029 (2016).

[33] B. G. Chen, B. Liu, A. A. Evans, J. Paulose, I. Cohen, V. Vitelli, and C. D. Santangelo, Topological Mechanics of Origami and Kirigami, Phys. Rev. Lett. 116, 135501 (2016).

[34] H. Abbaszadeh, A. Souslov, J. Paulose, H. Schomerus, and V. Vitelli, Sonic Landau Levels and Synthetic Gauge Fields 
in Mechanical Metamaterials, Phys. Rev. Lett. 119, 195502 (2017).

[35] R. Susstrunk and S. D. Huber, Observation of phononic helical edge states in a mechanical topological insulator, Science 349, 47 (2015).

[36] A. B. Khanikaev, R. Fleury, S. H. Mousavi, and A. Alù, Topologically robust sound propagation in an angular-momentumbiased graphene-like resonator lattice, Nat. Commun. 6, 8260 (2015).

[37] D. Rocklin, B. G. Chen, M. Falk, V. Vitelli, and T. C. Lubensky, Mechanical Weyl Modes in Topological Maxwell Lattices, Phys. Rev. Lett. 116, 135503 (2015).

[38] T. Kariyado and Y. Hatsugai, Manipulation of Dirac Cones in Mechanical Graphene, Sci. Rep. 5, 18107 (2015).

[39] D. Z. Rocklin, S. Zhou, K. Sun, and X. Mao, Transformable topological mechanical metamaterials, Nat. Commun. 8, 14201 (2017).

[40] M. Nash, D. Kleckner, A. Read, V. Vitelli, A. M. Turner, and W. T. M. Irvine, Topological mechanics of gyroscopic metamaterials, Proc. Natl. Acad. Sci. USA 112, 14495 (2015).

[41] M. Fruchart, Y. Zhou, and V. Vitelli, Dualities and non-Abelian mechanics, Nature (London) 577, 636 (2020).

[42] G. Baardink, A. Souslov, J. Paulose, and V. Vitelli, Localizing softness and stress along loops in 3D topological metamaterials, Proc. Natl. Acad. Sci. USA 115, 3 (2018).

[43] D. Bartolo and D. Carpentier, Topological Elasticity of Nonorientable Ribbons, Phys. Rev. X 9, 041058 (2019).

[44] A. Saremi and Z. Rocklin, Topological Elasticity of Flexible Structures, Phys. Rev. X 10, 011052 (2020).

[45] L. Zhang and X. Mao, Fracturing of topological Maxwell lattices, New J. Phys. 20, 063034 (2018).

[46] S. Ulrich, N. Upadhyaya, B. V. Opheusden, and V. Vitelli, Shear shocks in fragile networks, Proc. Natl. Acad. Sci. USA 110, 20929 (2013).

[47] E. Bogomolny, Stability of classical solutions, Sov. J. Nucl. Phys. 24, 449 (1976).

[48] T. Vachaspati, Kinks and Domain Walls: An Introduction to Classical and Quantum Solitons (Cambridge University Press, New York, 2006).

[49] A. Achúcarro, Vortices and flat directions: The uses of Bogomolny bounds, in Patterns of Symmetry Breaking, NATO Science Series, Ser. II, Mathematics, Physics, and Chemistry, Vol. 127 (Springer, Dordrecht, 2003), p. 273.

[50] E. Witten, Dynamical breaking of supersymmetry, Nucl. Phys. B 188, 513 (1981).

[51] E. Witten and D. I. Olive, Supersymmetry algebras that include topological charges, Phys. Lett. B 78, 97 (1978).

[52] E. Witten, Supersymmetry and Morse theory, J. Differ. Geom. 17, 661 (1982).
[53] T. Grover, D. N. Sheng, and A. Vishwanath, Emergent spacetime supersymmetry at the boundary of a topological phase, Science 344, 280 (2014).

[54] M. J. Lawler, Supersymmetry protected topological phases of isostatic lattices and kagome antiferromagnets, Phys. Rev. B 94, 165101 (2016).

[55] J. Attig, K. Roychowdhury, M. J. Lawler, and S. Trebst, Topological mechanics from supersymmetry, Phys. Rev. Res. 1, 032047 (2019).

[56] E. Demaine and J. O'Rourke, Geometric Folding Algorithms. Linkages, Origami, Polyhedra (Cambridge University Press, Cambridge, 2007).

[57] C. R. Calladine, Buckminster Fuller's “Tensegrity” structures and Clerk Maxwell's rules for the construction of stiff frames, Int. J. Solids Struct. 14, 161 (1978).

[58] A. J. Liu and S. R. Nagel, Jamming transition and the marginally jammed solid, Annu. Rev. Condens. Matter Phys. 1, 347 (2010).

[59] K. Sun and X. Mao, Continuum Theory for Topological Edge Soft Modes, Phys. Rev. Lett. 124, 207601 (2020).

[60] R. D. Kamien, The geometry of soft materials: A primer, Rev. Mod. Phys. 74, 953 (2002).

[61] K. Sun and X. Mao, Maxwell plates and phonon fractionalization, arXiv:1907.06620.

[62] The isostatic condition is exactly fulfilled by connecting the two ends of the chain (ring configuration). Once an edge is created, the eigenvalues of the rigidity matrix give rise to exactly one zero-energy mode which will remain localized to an edge of the chain.

[63] W. P. Su, J. R. Schrieffer, and A. J. Heeger, Solitons in Polyacetylene, Phys. Rev. Lett. 42, 1698 (1979).

[64] M. Shifman, Advanced Topics in Quantum Field Theory: A Lecture Course (Cambridge University Press, New York, 2012).

[65] A. R. Aguirre and G. F. Hidalgo, A supersymmetric exotic field theory in $(1+1)$ dimensions: One loop soliton quantum mass corrections, J. High Energy Phys. 12 (2018) 082.

[66] F. Cooper, A. Khare, and U. Sukhatme, Supersymmetry and quantum mechanics, Phys. Rep. 251, 267 (1995).

[67] R. Jackiw and J. R. Schrieffer, Solitons with fermion number $1 / 2$ in condensed matter and relativistic field theories, Nucl. Phys. B. 190, 253 (1981).

[68] R. Jackiw and C. Rebbi, Solitons with fermion number 1/2, Phys. Rev. D 13, 3398 (1976).

[69] R. Jackiw and P. Rossi, Zero modes of the vortex-fermion system, Nucl. Phys. B 190, 681 (1981).

[70] M. Nakahara, Geometry, Topology, and Physics (IOP Publishing, Bristol, 2003).

[71] See Supplemental Material at http://link.aps.org/supplemental/ 10.1103/PhysRevResearch.2.043098 for movies of topological mechanisms realized using LEGO. 